\title{
Charge-Density-Wave Origin of Dip-Hump Structures in the Tunnel Spectra of $\mathrm{Bi}_{2} \mathrm{Sr}_{2} \mathrm{CaCu}_{2} \mathrm{O}_{8+\delta}$
}

\author{
T. Ekino ${ }^{a}$, A.M. Gabovich ${ }^{b}$, Mai Suan Li $^{c}$, M. Peskala ${ }^{d}$, \\ H. SZYMCZAK ${ }^{c}$ AND A.I. VOITENKO ${ }^{b}$ \\ ${ }^{a}$ Hiroshima University, Faculty of Integrated Arts and Sciences \\ Higashi-Hiroshima, 739-8521, Japan \\ ${ }^{b}$ Institute of Physics, Nauki Ave. 46, 03028 Kiev, Ukraine \\ ${ }^{c}$ Institute of Physics, Polish Academy of Sciences \\ al. Lotników 32/46, 02-668 Warsaw, Poland \\ ${ }^{d}$ Department of Chemistry, Warsaw University \\ al. Żwirki i Wigury 101, 02-089 Warsaw, Poland
}

\begin{abstract}
We calculated the differential conductance $G$ as the function of the bias voltage $V$ across the tunnel junction between a normal metal or a conventional superconductor and an inhomogeneous superconductor with charge density waves. Spatial averaging over random domains with varying superconducting and normal state properties was carried out. For high- $T_{\mathrm{c}}$ oxides, irregularly distorted charge density wave patterns with spatially scattered values of various parameters were earlier shown to manifest themselves in a great body of experimental data. The results of calculation were applied to explain the well-known dip-hump structure in the $G(V)$ dependence for $\mathrm{Bi}_{2} \mathrm{Sr}_{2} \mathrm{CaCu}_{2} \mathrm{O}_{8+\delta}$ and other cuprates.
\end{abstract}

PACS numbers: 73.43.Jn, 71.45.Lr, 74.50.+r, 74.81.--g

\section{Introduction}

Tunnel quasiparticle differential conductance versus bias voltage dependences $G(V)=\mathrm{d} J / \mathrm{d} V$ (hereafter coined as current-voltage characteristics, CVCs) unveil characteristic gap-like features both for conventional Bardeen-CooperSchrieffer (BCS) superconductors and high- $T_{\mathrm{c}}$ oxides $[1,2]$. Here $J$ is the quasiparticle current, $V$ means voltage. For cuprates in addition to the superconducting gap $\Delta$, which is believed to have a $d$-wave symmetry, a pseudogap $\Pi$ of the disputed nature is often seen for many cuprates $[1,3]$. We think that the pseudogap is related to a phenomenon competing with the Cooper pairing, most probably being a charge density wave (CDW) instability $[4,5]$. CDW formation is accompanied by a destruction of the nested $(d)$ sections on the Fermi surface (FS), where the 
CDW-driven gap is developed. At the same time, a unique superconducting gap $\Delta$ appears both on $d$ and non-nested ( $n$ ) FS sections [6] (see below).

CVCs for high- $T_{\mathrm{c}}$ oxide junctions have a number of peculiarities. They are especially clearly seen in $\mathrm{Bi}_{2} \mathrm{Sr}_{2} \mathrm{CaCu}_{2} \mathrm{O}_{8+\delta}$ (BSCCO) $[2,7,8]$ that has become a testing ground for many ideas in theoretical superconductivity. In particular, CVCs for S-I-N junctions (S stands for a superconductor, I for an insulator, and $\mathrm{N}$ for a normal metal) are nonsymmetric with various amplitudes of the coherent peaks for branches for $V>0$ or $V<0$ and shapes of the so-called dip-hump structures (DHSs) - one per each branch - that are located at biases larger than the superconducting coherent peak positions. The loss of the CVC symmetry is also observed for junctions including other cuprates.

The majority of S-I-N junctions reveal a DHS only for one polarity of $V$. Nevertheless, there are also observed two symmetrically located DHSs (one per branch) but with amplitudes that can differ drastically [9]. The BCS-based theory of tunneling in superconducting junctions leads to symmetrical CVCs even if a junction is non-symmetrical. A lot of theories have been proposed to explain the above-mentioned nonsymmetricity, but the problem has not yet been resolved. The main trend is to explain the presence of the DHS as a manifestation of strong-coupling superconducting effects, conspicuous due to the quasiparticle interaction with a certain boson mode [9-11]. However, there is a lot of evidence (at least in BSCCO and related compositions) that the pseudogap $\Pi$ feature, which we attribute to the CDW, coexists with a superconducting gap below $T_{\mathrm{c}}$ within a wide doping range [3-5].

Therefore, we consider that the DHS occurs due to the overlap of the coherent peaks induced by the Cooper and pseudogap pairings. In this model, the hump is nothing else but a smeared peak caused by $\Pi$, while the dip is simply a transition region between those two peaks. Such an interpretation is directly supported by observations of periodic structures in BSCCO taking advantage of various experimental methods $[12,13]$. Photoemission studies reveal the $4 a_{0} \times 4 a_{0}$ charge ordered "checkerboard" state ( $a_{0}$ is a lattice constant) in $\mathrm{Ca}_{2-x} \mathrm{Na}_{x} \mathrm{CuO}_{2} \mathrm{Cl}_{2}$ [14]. A dynamical charge inhomogeneity probably connected to the stripe order has been recently observed in $\mathrm{La}_{2-x} \mathrm{Sr}_{x} \mathrm{CuO}_{4}$ (with $x=0.07,0.15$ ) [15].

We suppose $s$-symmetry for both the superconducting (SOP) and dielectric (DOP) order parameters (OPs) in agreement with the self-consistent theory of CDW superconductors (CDWSs) developed by us earlier [16] on the basis of the Bilbro-McMillan model [6], with both the gaps, superconducting $\Delta$ and dielectric $\Sigma$, having $s$-symmetry. In this work, only nonsymmetric $\mathrm{S}-\mathrm{I}-\mathrm{N}$ junctions are studied. Symmetrical superconductor-insulator-superconductor (S-I-S) junctions will be considered in a separate paper. 


\section{Theoretical background}

The detailed formulation of the self-consistent theory for a homogeneous partially gapped CDWS can be found elsewhere [16]. Here, we shall point out its fundamental features relevant to the subject concerned.

The FS of the ungapped homogeneous CDWS (i.e. above both superconducting-, $T_{\mathrm{c}}$, and CDW (structural-transition), $T_{d}$, critical temperatures) includes (i) two congruent ("nested", $i=1,2)$ sections, where the degenerate $(d)$ quasiparticle spectrum branches $\xi_{1,2}(p)$ reckoned from the common Fermi level are linked according to the relationship $\xi_{1}(\boldsymbol{p})=-\xi_{2}(\boldsymbol{p}+\boldsymbol{Q}), \boldsymbol{Q}$ being the CDW vector, and (ii) the remaining part $(i=3)$, where the quasiparticle spectrum $\xi_{3}(\boldsymbol{p})$ is non-degenerate $(n)$. The extent of such an FS partition is described by a parameter $\mu=N_{d}(0) /\left[N_{d}(0)+N_{n}(0)\right]$, where $N_{d}(0)$ and $N_{n}(0)$ are the quasiparticle densities of states (DOSs) at the $d$ and $n$ sections of the FS, respectively. The parameter $\mu$ by definition falls within the interval $0<\mu<1$. The CDWS Hamiltonian includes the interaction terms responsible for the dielectric and superconducting gappings of the FS. The superconducting gapping is a result of the BCS pairing and spans the whole FS.

Had the dielectric pairing constant been switched off, one would have obtained a "parent" BCS superconductor, characterized by the "bare" zero- $T$ order parameter $\Delta_{0}^{*}$, i.e. the corresponding critical temperature would have been $T_{\mathrm{c}}^{*}=\frac{\gamma}{\pi} \Delta_{0}^{*}(\gamma=1.7810 \ldots$ is the Euler constant, and the Boltzmann constant $\left.k_{\mathrm{B}}=1\right)$ and within the interval $0 \leq T \leq T_{\mathrm{c}}^{*}$ its OP $\Delta^{*}$ would have varied following the Mühlschlegel dependence $\Delta^{*}(T)=\Delta_{0}^{*} \operatorname{Mu}\left(T / T_{\mathrm{c}}^{*}\right)$, with $\operatorname{Mü}(0)=1$, a uniform gap $\Delta^{*}(T)$ being developed on the whole FS. On the other hand, if the constant of interaction that is responsible for the Cooper pairing had been switched off, we would have had, at $T<T_{d}^{*}$, a parent CDW-metal (CDWM) phase with the dielectric OP $\tilde{\Sigma}^{*}=\Sigma^{*} \mathrm{e}^{\mathrm{i} \varphi}$, characterized by the amplitude $\Sigma^{*}$ and the phase $\varphi$. The phase $\varphi$ is fixed by various factors both in the excitonic and Peierls scenarios, and acquires the values either 0 or $\pi$ in the first case or an arbitrary value in the Peierls state. The parameters $\mu, \Delta_{0}^{*}, \Sigma_{0}^{*}$, and $\varphi$ comprise a complete set of "bare" parameters to describe the CDWS.

In the framework of the self-consistent theory of the partially gapped CDWS, the superconducting and dielectric gappings can coexist only if the relationship $\Sigma_{0}^{*}>\Delta_{0}^{*}$ is satisfied [16]. Hence, as the temperature becomes lower, the CDWS undergoes first the dielectric (structural) phase transition at the actual $T_{d}$, which therefore coincides with $T_{d}^{*}\left(T_{d}^{*}=T_{d}\right)$. As $T$ continues to decrease, the CDWS behaves as its CDWM parent phase, i.e. the OP $\tilde{\Sigma}(T)=\tilde{\Sigma}_{0}^{*} \mathrm{Mu}\left(T / T_{d}\right)$ and the corresponding dielectric gap $|\tilde{\Sigma}(T)|$ appears on the $d$ sections of the FS until the actual superconducting critical temperature $T_{\mathrm{c}}<T_{d}$ is reached. For $0<T<T_{\mathrm{c}}$, the resulting self-consistent OP $\Delta$ behaves as $\Delta\left(T<T_{\mathrm{c}}\right)=\Delta_{0} \mathrm{Mu}\left(T / T_{\mathrm{c}}\right)$ with

$$
\Delta_{0}=\left(\Delta_{0}^{*} \Sigma_{0}^{*-\mu}\right)^{\frac{1}{1-\mu}}<\Delta_{0}^{*},
$$

and $T_{\mathrm{c}}=\frac{\gamma}{\pi} \Delta_{0}$, so that the unique gap $\Delta(T)$ develops on the whole FS [6]. Hence, 
the "combined" uniform gap

$$
D(T)=\sqrt{\Delta^{2}(T)+\Sigma^{2}(T)}
$$

appears on the $d$ FS sections and behaves as $D\left(T<T_{\mathrm{c}}\right)=\Sigma_{0}^{*} \mathrm{Mü}\left(T / T_{d}\right)$. It comprises a smooth continuation of $\Sigma(T)$ into the $T<T_{\mathrm{c}}$ region.

In what follows, the temperature Green functions (GFs) of the CDWS will be used as input quantities. To calculate quasiparticle tunnel currents only three of them $\mathcal{G}_{n}^{\mathrm{CDWS}}\left(\boldsymbol{p}, \omega_{n}\right), \mathcal{G}_{d}^{\mathrm{CDWS}}\left(\boldsymbol{p}, \omega_{n}\right)$, and $\mathcal{G}_{\mathrm{c}}^{\mathrm{CDWS}}\left(\boldsymbol{p}, \omega_{n}\right)$ are necessary [5], where $\omega_{n}=(2 n+1) \pi T, n=0, \pm 1, \pm 2, \ldots$ The first and the second GF $(n$ and $d)$ correspond to the quasiparticle propagation from the relevant FS section: $n$ or $d$. The Green function $\mathcal{G}_{\mathrm{c}}^{\mathrm{CDWS}}$ corresponds to the CDW pairing.

The quasiparticle tunnel current $J$ through the S-I-N junction between a homogeneous CDWS and a normal metal or a BCS superconductor is calculated according to the Larkin-Ovchinnikov approach [17]. In our case, it is a sum of three terms $J_{i}$,

$$
J(V)=\sum_{i=n, d, \mathrm{c}} J_{i}(V),
$$

of the same structure

$$
J_{i} \propto \frac{1}{R} \operatorname{Re} \int_{-\infty}^{\infty} \mathrm{d} \omega^{\prime} \int_{-\infty}^{\infty} \mathrm{d} \omega \frac{\operatorname{Im} G_{i}^{\mathrm{CDWS}}\left(\omega^{\prime}\right) G^{\mathrm{N}, \mathrm{BCS}}(\omega)}{\omega^{\prime}-\omega+e V+\mathrm{i} 0} .
$$

Here, $R$ is the tunnel resistance of the junction in the normal state, and $V \equiv$ $V_{\mathrm{N}}-V_{\mathrm{CDWS}}$ is the bias voltage across the junction reckoned from the potential of the CDWS electrode. Temporal Green's functions $G_{i}^{\mathrm{CDWS}}(\omega)$ can be obtained from $\mathcal{G}_{n}^{\mathrm{CDWS}}\left(\boldsymbol{p}, \omega_{n}\right), \mathcal{G}_{d}^{\mathrm{CDWS}}\left(\boldsymbol{p}, \omega_{n}\right)$, and $\mathcal{G}_{\mathrm{c}}^{\mathrm{CDWS}}\left(\boldsymbol{p}, \omega_{n}\right)$ by the well-known procedure [18].

The zero- $T$ CVC feature points of the quasiparticle current (3) through a CDWS-I-N (or CDWS-I-BCS) junction are located at biases $\mathrm{eV}= \pm \Delta$ and $\pm D$ [or $e V= \pm\left(\Delta+\Delta_{\mathrm{BCS}}\right)$ and $\left.\pm\left(D+\Delta_{\mathrm{BCS}}\right)\right]$. The main distinction from the $\mathrm{CVC}$ in the BCS-I-N case is the absence of the symmetricity with respect to the inversion of the bias voltage sign. The total current (3), the CDWS-I-N (or CDWS-I-BCS) junction is composed of three components: $J_{n}, J_{d}$, and $J_{\mathrm{c}}$. The symmetricity of the currents $J_{d}$ and $J_{n}$ is the same as for tunnel junctions involving normal metals and BCS-like superconductors, $J_{d, n}(-V)=-J_{d, n}(V)$, while $J_{\mathrm{c}}(-V)=J_{\mathrm{c}}(V)$. The anomalous behavior of the $J_{\mathrm{c}}$ component is connected to the dependence of the Green function $\mathcal{G}_{\mathrm{c}}^{\mathrm{CDWS}}\left(\boldsymbol{p}, \omega_{n}\right)$ on $\Sigma$ rather than on $\Sigma^{2}$. The summation of components makes the total $J(V)$ and the corresponding $G(V)$ nonsymmetric.

The CVC peculiarities of the $J_{d}$ and $J_{\mathrm{c}}$ components at $\mathrm{eV}= \pm D$ [or $\left.e V= \pm\left(D+\Delta_{\mathrm{BCS}}\right)\right]$ can either enhance or compensate each other. In the latter case, the logarithmic singularity can even be transformed into a cusp (in the limit $\left.\Delta, \Delta_{\mathrm{BCS}} \rightarrow 0\right)$. The nonsymmetricity of $G(V)$ for tunnel junctions involving CDWSs constitutes one of the key points of the proposed theory. 
The second key point of our theory is connected with the intrinsic nonhomogeneity of the CDWS state in high- $T_{\mathrm{c}}$ cuprates, which is inherent even to the best BSCCO single-crystal specimens. First of all, we would like to point at regular modulated structures of charge distribution in BSCCO, including stripe-like and checkerboard ones, which were found in a number of experiments (see, e.g., Refs. [12-15, 19]). Such modulated structures remarkably resemble CDWs, and their appearance even correlates with that of the pseudogap [19].

At the same time, it is well known that $\Delta$ and $\Pi$ values are distributed over the cuprate samples' surfaces in a patch-like irregular manner. We insist that small energy gaps with large and narrow coherence peaks and larger gaps with lower and broadened peaks have different nature and are generated by superconducting and CDW OPs, although many authors consider both types of gaps as associated with superconductivity.

\section{Results of calculation}

Each of the model parameters may have a certain dispersion, and all the four can be scattered concurrently, in accordance to what happens in cuprates. But it would be very difficult to analyze the results of such calculations. Therefore, we shall consider the variation of each parameter separately to trace the tendency which such a variation might bring about. Each control parameter $x$ was regarded scattered within the interval $\left[x_{0}-\sigma, x_{0}+\sigma\right]$ around the mean value $x_{0}$, according to the Gauss probability distribution law; $\sigma$ is a dispersion. The current $J(V)$ was averaged over this distribution. This procedure was followed by the numerical differentiation over $V$ with an arbitrarily chosen differentiation interval $\delta V$, which gave us the conductance spectra $G(V)=R \mathrm{~d} J / \mathrm{d} V$. For sufficiently small $\delta V$ the results were weakly dependent on $\delta V$. Therefore, throughout the paper we used the value $\delta V= \pm 2.5 \mathrm{meV}$. One should note that such kind of a numerical calculation reasonably well mimics the experimental results obtained from the $J(V)$ raw data by their differentiation.

First, we consider the dispersion of the bare SOP $\Delta_{0}^{*}$. The corresponding dependences $G(V)$ are shown in Fig. 1a for a BSCCO-I- $\mathrm{V}_{3} \mathrm{Si}$ junction. The phase $\varphi$ is chosen equal to $\pi$ to fit the experimental CVCs $[2,7]$, when the DHS reveals itself for negative $V$. One sees that the $D$-driven singularities remained almost intact while averaging, and no structures similar to the dip-hump one are observed. Therefore, the dispersion of the $\Delta_{0}^{*}$ parameter alone turns out insufficient to explain the experimental data.

The results of calculations resemble the observed $G(V)$ dependences for BSCCO much more if it is the parameter $\Sigma_{0}^{*}$ that is assumed to scatter (Fig. 1b). Indeed, according to our basic equations, all the four CVC peculiarities $\mathrm{eV}=$ $\pm\left(\Delta+\Delta_{\mathrm{BCS}}\right)$ and $\pm\left(D+\Delta_{\mathrm{BCS}}\right)$ become smeared, although to the various extent: the large singularities $e V= \pm\left(\Delta+\Delta_{\mathrm{BCS}}\right)$ almost preserve their shape, the large singularity $e V=-\left(D+\Delta_{\mathrm{BCS}}\right)$ transforms into a DHS, and the small one $e V=\left(D+\Delta_{\mathrm{BCS}}\right)$ disappears on the scale selected. The one-polarity character of 
the DHS appropriate to experimental CVCs [2] is reproduced excellently. Owing to the relationship (1), the actual parameter $\Delta$ also scatters, but, due to the small value of $\mu$, this fluctuation becomes too small to be observed in the plot.

In Fig. 2 the dependences $G(V)$ are displayed for a BSCCO-I-N tunnel junction. As is well known, the type of the $\Delta$-related singularity changes from
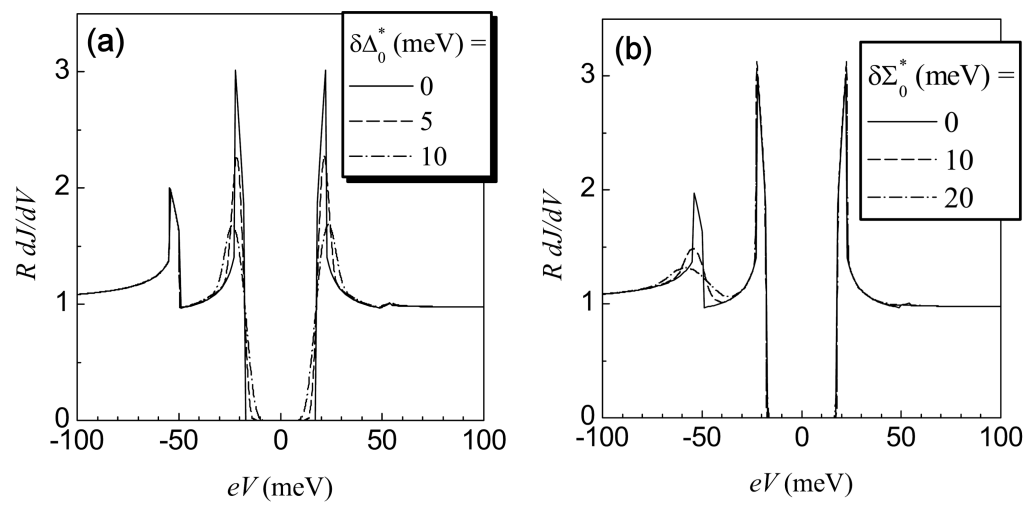

Fig. 1. (a) Dependences of the tunnel conductance $G$ on voltage $V$ for the junction $\mathrm{Bi}_{2} \mathrm{Sr}_{2} \mathrm{CaCu}_{2} \mathrm{O}_{8+\delta}$ (BSCCO) $-\mathrm{I}-\mathrm{V}_{3} \mathrm{Si}$ at a temperature of $T=4.2 \mathrm{~K}$. I symbolizes insulator. Relevant parameters of the BSCCO are as follows: the bare dielectric and superconducting gaps $\Sigma_{0}^{*}=50 \mathrm{meV}, \Delta_{0}^{*}=50 \mathrm{meV}$, the dielectric gapping parameter $\mu=0.1$, the phase of the dielectric order parameter $\varphi=\pi$. The superconducting gap of $\mathrm{V}_{3} \mathrm{Si}$ at $T=0$ is $\Delta_{\mathrm{BCS}}=2.3 \mathrm{meV}$. The dispersion $\delta \Delta_{0}^{*}$ of $\Delta_{0}^{*}$ is shown in the legend. (b) The same as in part (a) but with the spread $\delta \Sigma_{0}^{*}$ of $\Sigma_{0}^{*}$.

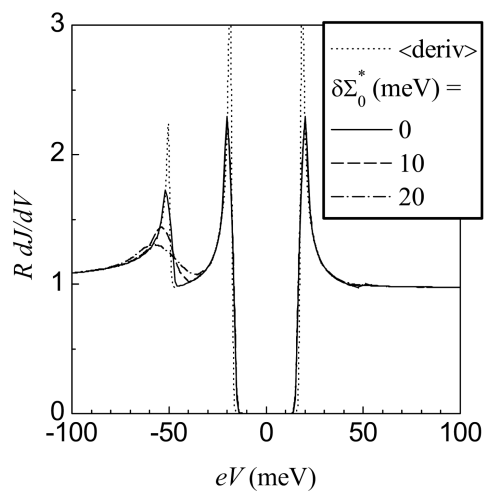

Fig. 2. The same as in Fig. $1 \mathrm{~b}$ but for the junction BSCCO-I-N, where N stands for a normal metal. The dotted curve ( $\langle$ deriv $\rangle)$ was numerically calculated not directly from integral (4) with the subsequent differentiation, as the rest of the curves in all figures, but by the analytical differentiation of Eq. (4) with the subsequent numerical integration. Comparison with the experimental data shows that the so-calculated $\langle$ deriv $\rangle$-curve overestimates the gap-related features of $G(V)$. 
the steep jump for an S-I-S' sandwich, where $\mathrm{S}^{\prime}$ stands for a superconductor different from S, into a square-root peculiarity for an S-I-N one. The same is true for our case when a CDWS electrode is inserted instead of an $\mathrm{S}$ one. The dotted curve denotes an unaveraged dependence obtained by a direct numerical calculation of the conductance $G(V)$, which in its turn had been found after a preliminary differentiation of Eq. (4) before its integration. All other dependences were found by a differentiation of the numerically calculated integral (4). As we have already mentioned, such a method is a better fit to the usual procedure of getting $G(V)$ from the $J(V)$ measurements. The solid curve corresponds to the absence of any CDW gap scatter, whereas next two dependences show how the sample nonhomogeneity may distort the intrinsic square-root singularity at $e V=-D$ and convert it into a DHS. It is exactly what is observed in experiments $[2,7,8]$. One should underscore that a DHS position is governed by the DOP phase $\varphi$, which is thus a fitting parameter. If $\varphi=\pi / 2$ or in the absence of the preferred surface DOP phase, DHSs would reveal themselves symmetrically for both $V$ polarities.

It is important to recognize that experimental CVCs demonstrate features appropriate both to averaging over $\Delta_{0}^{*}$ (Fig. 1a) and $\Sigma_{0}^{*}$ (Figs. 1b and 2).

\section{Conclusions}

Thus, assuming the CDW origin of the DHS in tunnel spectra of high- $T_{\mathrm{C}}$ oxides, in particular, BSCCO, made it possible to qualitatively describe the CVCs. On the other hand, the widely spread alternative explanation [11] of the DHS, which is based on the existence of a certain boson mode of an unknown nature with the energy $\Omega$, cannot account for the observed non-symmetrical form of the CVCs. In addition, a wide-range oxygen doping of BSCCO leads to the shift of both the $\Delta$ and DHS locations, which are not linked with each other by any simple relationship [20]. At the same time, in the framework of the boson-based approach the DHS in the S-I-N junctions should be positioned at $e V=\Delta+\Omega$ in S-I-N and $e V=2 \Delta+\Omega$ in S-I-S junctions.

In our scenario, the locations of $\Delta$ and DHS are also correlated, which is traced while doping the material. Nevertheless, the link between two quantities is rather complicated, because the $D$-peculiarity position depends on the doping-driven shift of $T_{d}$ in a twofold non-linear way: through $\Sigma$ and $\Delta$. The latter quantity, in its turn, also depends on $\Sigma$. The above calculations show the inaccuracy of the conclusion [10] that a strong observed dip cannot be described under the assumption of its non-superconducting nature. It should be noted that our conclusions, although being obtained assuming the $s$-wave symmetry of both OPs, are quite general and applicable to the situation when their symmetry is reduced (e.g., for the $d$-wave symmetry).

\section{Acknowledgments}

A.M.G. is grateful to Kasa im. Józefa Mianowskiego and Fundacja na Rzecz Nauki Polskiej for the financial support of his visit to Warsaw and to 
Maciej Maśka (Institute of Physics, University of Silesia, Katowice, Poland) and Karol Wysokiński (Institute of Physics and Nanotechnology Centre, M. Curie-Skłodowska University, Lublin, Poland) for fruitful discussions.

\section{References}

[1] T. Ekino, Y. Sezaki, H. Fujii, Phys. Rev. B 60, 6916 (1999).

[2] N. Miyakawa, J.F. Zasadzinski, L. Ozyuzer, P. Guptasarma, D.G. Hinks, C. Kendziora, K.E. Gray, Phys. Rev. Lett. 83, 1018 (1999).

[3] Y. Yamada, M. Suzuki, Phys. Rev. B 74, 054508 (2006).

[4] A.M. Gabovich, A.I. Voitenko, J.F. Annett, M. Ausloos, Supercond. Sci. Technol. 14, R1 (2001).

[5] A.M. Gabovich, A.I. Voitenko, M. Ausloos, Phys. Rep. 367, 583 (2002).

[6] G. Bilbro, W.L. McMillan, Phys. Rev. B 14, 1887 (1976).

[7] Ch. Renner, B. Revaz, J.-Y. Genoud, K. Kadowaki, Ø. Fischer, Phys. Rev. Lett. 80, 149 (1998).

[8] J. Zasadzinski, L. Ozyuzer, N. Miyakawa, D.G. Hinks, K.E. Gray, Physica C 341-348, 867 (2000).

[9] Y. DeWilde, N. Miyakawa, P. Guptasarma, M. Iavarone, L. Ozyuzer, J.F. Zasadzinski, P. Romano, D.G. Hinks, C. Kendziora, G.W. Crabtree, K.E. Gray, Phys. Rev. Lett. 80, 153 (1998).

[10] P. Romano, L. Ozyuzer, Z. Yusof, C. Kurter, J.F. Zasadzinski, Phys. Rev. B 73, 092514 (2006).

[11] J.F. Zasadzinski, L. Ozyuzer, L. Coffey, K.E. Gray, D.G. Hinks, C. Kendziora, Phys. Rev. Lett. 96, 017004 (2006).

[12] S.A. Kivelson, I.P. Bindloss, E. Fradkin, V. Oganesyan, J.M. Tranquada, A. Kapitulnik, C. Howald, Rev. Mod. Phys. 75, 1201 (2003).

[13] J. Lee, K. Fujita, K. McElroy, J.A. Slezak, M. Wang, Y. Aiura, H. Bando, M. Ishikado, T. Masui, J.-X. Zhu, A.V. Balatsky, H. Eisaki, S. Uchida, J.C. Davis, Nature 442, 546 (2006).

[14] K.M. Shen, F. Ronning, D.H. Lu, F. Baumberger, N.J.C. Ingle, W.S. Lee, W. Meevasana, Y. Kohsaka, M. Azuma, M. Takano, H. Takagi, Z.-X. Shen, Science 307, 901 (2005).

[15] D. Reznik, L. Pintschovius, M. Ito, S. Iikubo, M. Sato, H. Goka, M. Fujita, K. Yamada, G.D. Gu, J.M. Tranquada, Nature 440, 1170 (2006).

[16] A.M. Gabovich, M.S. Li, H. Szymczak, A.I. Voitenko, J. Phys., Condens. Matter 15, 2745 (2003).

[17] A.I. Larkin, Yu.N. Ovchinnikov, Zh. Eksp. Teor. Fiz. 51, 1535 (1966).

[18] A.M. Gabovich, A.I. Voitenko, J. Phys., Condens. Matter 9, 3901 (1997).

[19] M. Vershinin, S. Misra, S. Ono, Y.A.Y. Ando, A. Yazdani, Science 303, 1995 (2004).

[20] J.F. Zasadzinski, L. Ozyuzer, N. Miyakawa, K.E. Gray, D.G. Hinks, C. Kendziora, J. Phys. Chem. Solids 63, 2247 (2002). 